\title{
Human and Animal RNA Virus Diversity Detected by Metagenomics in Cameroonian Clams
}

\author{
Patrice Bonny 1,2,3, Julien Schaeffer ${ }^{1}$, Alban Besnard ${ }^{1}$, Marion Desdouits', \\ Jean Justin Essia Ngang ${ }^{2}$ and Françoise S. Le Guyader ${ }^{1 \star}$
}

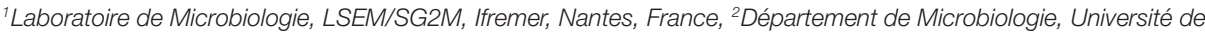
Yaoundé I, Yaoundé, Cameroon, ${ }^{3}$ Centre de Recherche en Alimentation et Nutrition, IMPM, Yaoundé, Cameroon

\section{OPEN ACCESS}

Edited by:

Erna Geessien Kroon, Federal University of Minas Gerais,

Brazil

Reviewed by:

Jemma Geoghegan,

University of Otago, New Zealand

Jesus L. Romalde,

University of Santiago

de Compostela, Spain

*Correspondence:

Françoise S. Le Guyader soizick.le.guyader@ifremer.fr

Specialty section:

This article was submitted to

Virology,

a section of the journal

Frontiers in Microbiology

Received: 03 September 2021

Accepted: 18 October 2021

Published: 30 November 2021

Citation:

Bonny P, Schaeffer J, Besnard A, Desdouits M, Essia Ngang JJ and Le Guyader FS (2021) Human and Animal RNA Virus Diversity Detected

by Metagenomics in Cameroonian

Clams.

Front. Microbiol. 12:770385. doi: 10.3389/fmicb.2021.770385
Many recent pandemics have been recognized as zoonotic viral diseases. While their origins remain frequently unknown, environmental contamination may play an important role in emergence. Thus, being able to describe the viral diversity in environmental samples contributes to understand the key issues in zoonotic transmission. This work describes the use of a metagenomic approach to assess the diversity of eukaryotic RNA viruses in river clams and identify sequences from human or potentially zoonotic viruses. Clam samples collected over 2 years were first screened for the presence of norovirus to verify human contamination. Selected samples were analyzed using metagenomics, including a capture of sequences from viral families infecting vertebrates (VirCapSeq-VERT) before Illumina NovaSeq sequencing. The bioinformatics analysis included pooling of data from triplicates, quality filtering, elimination of bacterial and host sequences, and a deduplication step before de novo assembly. After taxonomic assignment, the viral fraction represented $0.8-15 \%$ of reads with most sequences (68-87\%) remaining un-assigned. Yet, several mammalian RNA viruses were identified. Contigs identified as belonging to the Astroviridae were the most abundant, with some nearly complete genomes of bastrovirus identified. Picobirnaviridae sequences were related to strains infecting bats, and few others to strains infecting humans or other hosts. Hepeviridae sequences were mostly related to strains detected in sponge samples but also strains from swine samples. For Caliciviridae and Picornaviridae, most of identified sequences were related to strains infecting bats, with few sequences close to human norovirus, picornavirus, and genogroup $V$ hepatitis $A$ virus. Despite a need to improve the sensitivity of our method, this study describes a large diversity of RNA virus sequences from clam samples. To describe all viral contaminants in this type of food, and being able to identify the host infected by viral sequences detected, may help to understand some zoonotic transmission events and alert health authorities of possible emergence.

Keywords: shellfish, metagenomics, human enteric viruses, mammal viruses, zoonosis 


\section{INTRODUCTION}

In the last few years, Next Generation Sequencing (NGS) has been increasingly used to study microbial populations in environmental samples. This approach allows the description of all nucleic acid sequences present in a sample and thus, theoretically, enables the identification of all organisms including viruses (Dance, 2021). As a catch-all agnostic approach, it holds great promise for monitoring and determining viral diversity in food and environmental samples such as sewage and shellfish (Desdouits et al., 2020; Nieuwenhuijse et al., 2020). Metagenomic sequencing has been applied to estimate viral diversity in different ocean waters, and sewage samples across the globe, showing its interest to describe viral communities (Nieuwenhuijse et al., 2020; Sunagawa et al., 2020).

Many recent human pandemics have been recognized as zoonotic viral diseases. If, in some cases, the origin has been linked to the modification of wildlife habitats or food consumption, many causes or sources are still unknown (Devaux et al., 2019; Osterhauss et al., 2020). Among main hypotheses, environment plays an important role as it can be the link between humans and the original host. Being able to describe the viral diversity in environmental samples may constitute the first step to understand the key issues in zoonotic transmission and help its prevention. However, the strategy of environmental sampling represents a major challenge. For human contaminations, sewage sampling is clearly a good approach, but when the animal or wildlife excreta has to be considered, other approaches are necessary (Nieuwenhuijse et al., 2020; Osterhauss et al., 2020).

The demand for shellfish is increasing worldwide given their nutritive value or taste. In addition, they grow without human intervention and constitute a substantial source of nutrients for many populations (Venugopal and Gopakumar, 2018). However, they have been identified for many years as highrisk food (Metcalf et al., 1995). Indeed, because of their mode of feeding by filtration of large volumes of water, they can concentrate various types of contaminants from their environment, including foodborne viruses. In line with this, many enteric viruses, such as noroviruses, enteroviruses, or hepatitis viruses, can be detected in shellfish tissues following their exposure to human sewage (La Rosa et al., 2017; Strubbia et al., 2019). In some cases, during shellfish-borne outbreaks, the high viral diversity in shellfish makes it difficult to identify the exact etiological agent, leading to an erroneous identification if the detection approach is too selective (Nieuwenhuijse and Koopmans, 2017), thereby explaining the need for a holistic approach when detecting viruses in shellfish. This also suggests that we can use shellfish as environmental sentinel, since they filter and concentrate the different pathogens present in the environment (Polo et al., 2021).

In West Africa, Galatea paradoxa is a freshwater clam found in several rivers including the Sanaga river and constitutes a substantial source of protein for local populations as well as a significant economic income (Ajonina et al., 2005). However, like all shellfish around the world, they are prone to accumulate various contaminants, including human enteric viruses. Our previous work, on the same site, reported Sanaga clams contamination with norovirus and hepatitis A virus, suggesting contamination by human sewage (Bonny et al., 2020). Furthermore, a sequence of hepatitis A virus close to the genotype $\mathrm{V}$ known to infect non-human primates was also detected. These findings demonstrate the contamination of these clams by both human and animal viruses, suggesting that they can reflect the diversity of viruses circulating in the Sanaga River growing site.

To deeply investigate the viral contamination, we performed additional sampling and applied a metagenomic approach to assess the diversity of RNA viral sequences of strains that may infect vertebrates in these clams and identify potentially pathogenic viruses, both humans and potentially zoonotic.

\section{MATERIALS AND METHODS}

\section{Sample Collection}

Two sampling points (Bol and $\mathrm{Mou}$ ) were selected on the Sanaga river (Cameroon, Central Africa), $12 \mathrm{~km}$ upstream the Atlantic Ocean (Figure 1) and located in a subdivision of about 15,000 inhabitants (non-official estimation performed in 2016/personal communication). These two points were distant of about $2.5 \mathrm{~km}$ and located on the same side of the river. Naturally growing clams Galatea paradoxa were collected monthly in each point between February 2018 and February 2020 (except on one occasion for site Bol and three occasions for site Mou). Each sample was constituted of 10-15 alive individuals. After rapid cleaning under tap water, clams were frozen $\left(-20^{\circ} \mathrm{C}\right)$ for further shipping to the Ifremer laboratory in France.

\section{Sample Preparation, Norovirus, and Hepatitis A Virus Detection}

On arrival, samples were thawed, shucked, and dissected. Digestive tissues (DT) were recovered, pooled, chopped, distributed in $2 \mathrm{~g}$ aliquots, and immediately stored at $-20^{\circ} \mathrm{C}$ until analysis. Viruses were eluted from DT using proteinase K method (ISO, 15216-1, 2017), and nucleic acids were extracted using the NucliSENS kit (bioMérieux, France). After validation of quality controls (extraction efficiency and inhibitor removal), the quantitative detection of norovirus and hepatitis A virus was performed by RT-qPCR (ISO, 15216-1, 2017). All samples were analyzed in the triplicates of undiluted nucleic acids using a one-step real-time RT-PCR kit (Invitrogen) and a MX3000P Quantitative PCR system (Agilent Technologies France).

\section{Sample Preparation for Metagenomic Analysis}

Samples positive for norovirus and hepatitis A virus were submitted to a novel extraction using an optimized protocol set up for metagenomics analysis (Strubbia et al., 2020). Briefly DT aliquots $(2 \mathrm{~g})$ were incubated with proteinase $\mathrm{K}$ solution (30 U/mg, Sigma-Aldricht, St-Quentin France) for $15 \mathrm{~min}$ at $37^{\circ} \mathrm{C}$ under agitation (32 rotations $/ \mathrm{min}$ ), and $15 \mathrm{~min}$ at $60^{\circ} \mathrm{C}$ The mixture was sonicated (Bandelin UD 2200 with cup-horn 


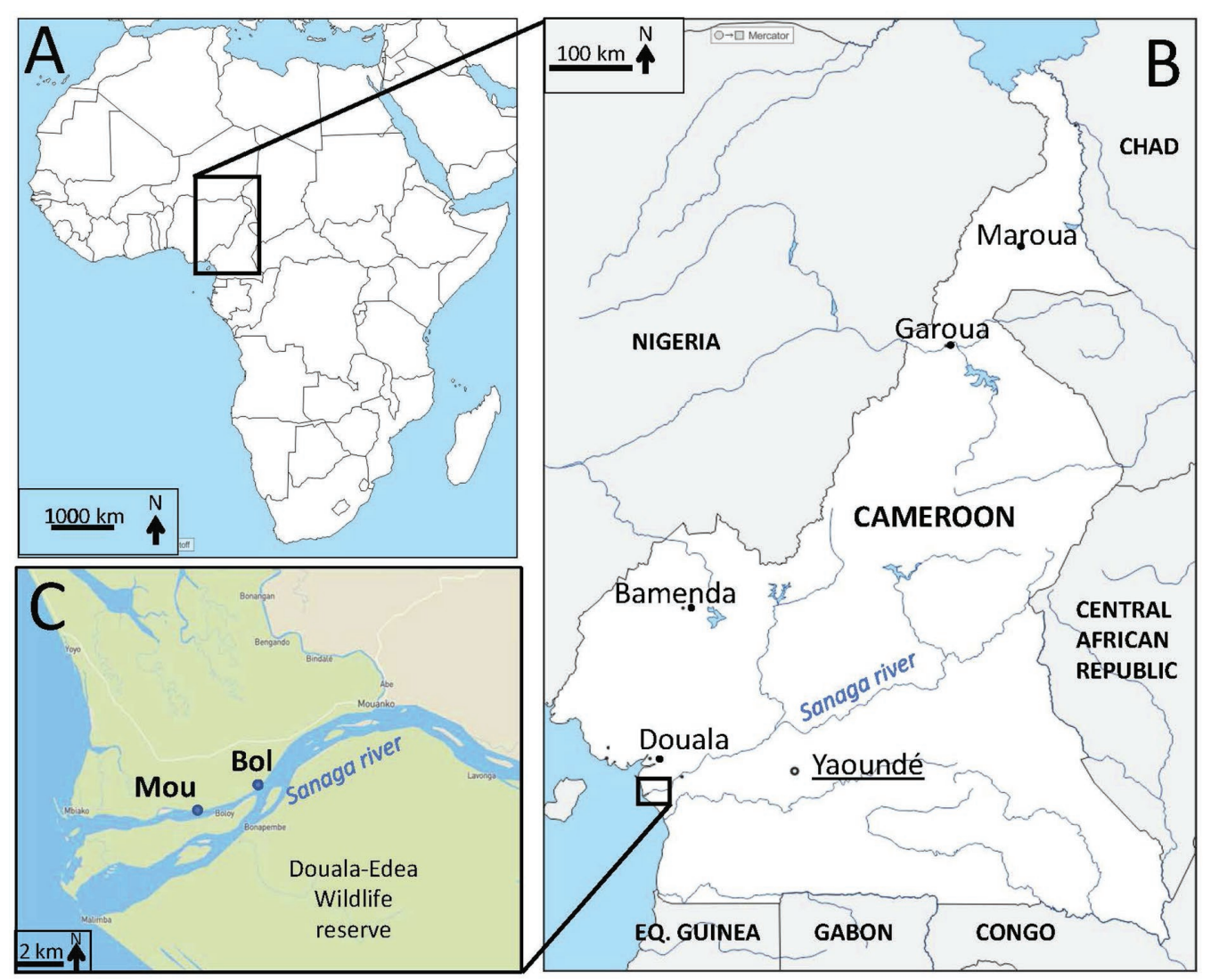

FIGURE 1 | Localization of sampling sites. (A) Map of Africa (d-maps.com) with Cameroon in the central region. (B) Map (d-maps.com) of Cameroon (white) with frontiers (black lines), neighboring countries (grey), capital (black circle, underlined) and main cities (black dots), main rivers and lakes (blue) including the Sanaga river. Eq. Guinea=Equatorial Guinea. (C) Zoom on the Sanaga river estuary with the location of study sampling sites (Bol, Mou) on the Sanaga river in the DoualaEdéa wildlife reserve (light green).

adaptor) for $3 \mathrm{~min}$ at maximum power $(100 \%$; cycles of $1 \mathrm{~min}$ with $1 \mathrm{~min}$ on ice) and centrifuged for $5 \mathrm{~min}$ at $3,000 \times \mathrm{g}$. Supernatant was measured, transferred to a $15-\mathrm{ml}$ tube, mixed with sodium pyrophosphate ( $10 \mathrm{mM}$ final concentration), and incubated for $40 \mathrm{~min}$ at $4^{\circ} \mathrm{C}$ under gentle agitation. After centrifugation for $20 \mathrm{~min}$ at $8000 \times g$, supernatant (approximately $3 \mathrm{ml}$ ) was recovered, mixed with $1.5 \mathrm{ml}$ of polyethylene glycol 6000 (PEG 24\% wt/vol, Sigma-Aldrich)-sodium chloride (1.2 M), and incubated for $1 \mathrm{~h}$ at $4^{\circ} \mathrm{C}$. After centrifugation for $20 \mathrm{~min}$ at $10,000 \times g$, the pellet was resuspended in $2 \mathrm{ml}$ of preheated $\left(56^{\circ} \mathrm{C}\right)$ glycine buffer $(0.05 \mathrm{M} ; \mathrm{pH} 9)$, then filtered using 5 , 1.5 , and $0.45 \mu \mathrm{m}$ acetate cellulose filters. Filtrate was treated with $20 \mu \mathrm{l}$ of OmniCleave ${ }^{\mathrm{TM}}$ Endonuclease (Lucigen corporation; $200 \mathrm{U} / \mu \mathrm{l})$ and $200 \mu \mathrm{l}$ of $\mathrm{MgCl}_{2}(100 \mathrm{mM})$ for $1 \mathrm{~h}$ at $37^{\circ} \mathrm{C}$.

The extraction of nucleic acids was carried out using lysis buffer and the NucliSENS kit (bioMerieux) with the semiautomatized eGENE-UPTM system (bioMerieux). Nucleic acids were treated by TURBO DNase $(25 \mathrm{U})$ for $30 \mathrm{~min}$ at $37^{\circ} \mathrm{C}$ (Ambion, Thermo Fischer Scientific, France). An additional RNA purification was carried out using the RNA Clean Concentrator ${ }^{\mathrm{TM}}-5$ kit (Zymo Research, Irvine, United States). Nucleic acids were recovered in $100 \mu$ l of elution buffer and stored at $-80^{\circ} \mathrm{C}$.
The detection of norovirus and hepatitis A virus was verified as described above, before libraries preparation.

\section{Library Preparation and Metagenomic Sequencing}

Technical triplicates were performed for cDNA reversetranscription of RNA extracts using the enzyme Superscript II (Thermofisher, France) and random hexamer primers (Thermofisher, France). After the production of cDNA using the second strand reaction buffer and synthesis enzyme mix (NEBNext Ultra RNA Library prep, New England Biolabs, France), a physical fragmentation (Ultrasonicator M220, Covaris) was carried out for $110 \mathrm{~s}$ (Strubbia et al., 2020).

Libraries were prepared using the KAPA Prep Kit (Roche, France). After ligation of the adapters, several libraries cleanup steps using AmPure XP Beads (Beckman Coulter, United States), and $80 \%$ ethanol were carried out to select fragments between 150 and $500 \mathrm{bp}$. The libraries were then quantified and pooled equimolarly into seven pools, after which viral enrichment was performed using the VirCapSeq-VERT capture panel (Roche, France) (Wylie et al., 2015). 
The sequencing was carried out on the Illumina NovaSeq 6000 using NovaSeq reagent Kit to generate $2 \times 250$ base pair reads at the ICM platform (Institut Cerveau Moelle, Paris, France).

\section{Bioinformatic Pipeline}

Bioinformatic analysis was performed using nextflow to monitor the run on the computing cluster. Fastq files from technical triplicates were pooled at the beginning of the analysis. TrimGalore was used for quality filtering and adapter removal, with a quality threshold of 25 . Then, a second filtering of the non-target reads, in this case non-viral reads such as host or bacterial rRNA, was carried out by mapping clean reads using Bowtie2 (very-sensitive option) (Langmead and Salzberg, 2012) on the Silva database (Nooij et al., 2018). Finally, duplicate reads were binned using cd-hit-dup with a prefix length of 50 for each read (Fu et al., 2012), in order to simplify the data set, facilitate the assembly step, and reduce PCR bias during abundance calculation.

Taxonomic assignation of the reads was done using Kaiju with the nr_euk database (February, 2020), on the raw data and on the data post deduplication to assess the composition of our samples and impact of preprocess steps (filtering, rRNA removal, and deduplication).

A de novo assembly was performed using metaSPAdes with kmer lengths 21, 33, 55, 77, and 99 (Nurk et al., 2017). Contigs longer than $300 \mathrm{bp}$ were identified using BLASTn 2.6.0 (e value 1E-5) on the nt NCBI bank (Camacho et al., 2009). In the absence of a match, the search was repeated using DIAMOND (e value 1E-3) on the nr NCBI bank (Buchfink et al., 2015). To evaluate the coverage of each contig, post-process reads were mapped using Bowtie 2 on the metaSPAdes contigs. Multimapped reads were removed as a source of potential overestimation of the abundance. Taxonomic identification was done using Entrez direct tool, the taxid allowing to extract information at a defined taxonomic level. Families were determined on the krona file taking all families that account for more than $0.01 \%$ of the viral reads in any of our samples.

Reads per millions (rpm) were calculated as the number of reads per family divided by the total number of trimmed and deduplicated reads, using $\mathrm{R}$, and heatmaps were generated using the ggplot $\mathrm{R}$ package. For the phylogenetic analysis, amino acid alignments were performed using Muscle and Clustal Omega. Phylogenetic trees were constructed using IQtree with a bootstrap of 1,000 .

\section{RESULTS}

\section{Norovirus and Hepatitis A Virus Detection in Samples}

All 45 collected samples yielded extraction efficiencies above $1 \%$ and none presented PCR inhibitors (ISO, 15216-1, 2017). A total of 31 samples were positive for norovirus GI and/or GII (Table 1). The same number of samples was collected from each site, and no difference in terms of prevalence or genomic concentrations was detected for norovirus contamination
TABLE 1 | Detection and quantification of norovirus and hepatitis A in collected samples.

\begin{tabular}{|c|c|c|c|c|c|c|}
\hline \multirow{2}{*}{ Site } & \multirow{2}{*}{$\begin{array}{c}\text { Nb of } \\
\text { sample }\end{array}$} & \multirow{2}{*}{$\begin{array}{c}\text { Ext. eff." } \\
(\%)\end{array}$} & \multicolumn{2}{|c|}{ Norovirus } & \multicolumn{2}{|c|}{ Hepatitis A virus } \\
\hline & & & Nb pos & $\mathrm{GMC}^{\#}$ & Nb pos & GMC $^{\#}$ \\
\hline Bol & 23 & $13(3-67)$ & 15 & 563 & 6 & 69 \\
\hline Mou & 22 & $9(4-40)$ & 16 & 297 & 10 & 75 \\
\hline Total & 45 & $11(3-67)$ & 31 & 405 & 16 & 73 \\
\hline
\end{tabular}

"Ext eff: mean extraction efficiency calculated for samples collected on each site and total samples, expressed as $\%$, with the range of extraction efficiency obtained into brackets 0 .

"GMC: geometric mean concentration calculated using concentrations obtained for each positive sample and expressed as cRNA/g of clam digestive tissue.

between sites (Table 1). Regarding hepatitis A virus contamination, only six samples were detected positive on site Bol compared to 10 samples on site Mou, with low concentrations very close to the limit of quantification.

To gain insight into the diversity of these viral pathogens, we selected 22 samples (13 from site Bol and nine from site Mou) collected during different months for metagenomic analyzes.

\section{Metagenomic Data}

The Illumina sequencing generated approximately 1.9 billion raw reads ranging from 42 to 177 million per library. Of these, $20 \%$ were removed by quality filtering and rRNA removal (Table 2).

To reduce the data size and optimize computing time for de novo assembly, the dataset was simplified by removing duplicate reads (deduplication), resulting in up to $85 \%$ of eliminated reads for some libraries. To verify the possible impact on reads distribution between the different life kingdoms, we performed a fast taxonomic assignation using Kaiju before and after the deduplication step (Figure 2). The kingdom that was most affected by deduplication was Bacteria with $88-96 \%$ of removed reads and Eukaryota with a fraction of removed reads between 77 and $95 \%$. The number of reads for the viral fraction was reduced by $79-83 \%$ following deduplication with few variations between samples. Eventually, $13.8 \%$ of initial reads (264 millions) were retained after the deduplication step. Of these, $68-87 \%$ were unclassified, $7.1-21.4 \%$ were assigned as bacteria, $0.7-10.2 \%$ as Eukaryote, $1.1-7.5 \%$ as virus, and $0.1-0.7 \%$ as Archaea (Figure 2B). All the following analyses were performed on deduplicated reads.

\section{Metagenome Assembly}

MetaSPAdes assembled $77 \%$ of deduplicated reads into a total of 268,378 contigs, of which 204,107 were longer than 300 base pairs. Of these, 98,476 (48\%) contigs were annotated by either BLASTn or DIAMOND (Table 2).

A total of 198 millions of reads were assigned using BLAST, 95 millions of which being identified as viral sequences (Table 2). For each sample (three pooled libraries), the percentage of reads identified as viral sequences varied from 
TABLE 2 | Summary of read numbers and contigs at different steps of the pipeline per sample.

\begin{tabular}{|c|c|c|c|c|c|c|c|c|c|c|}
\hline \multirow[b]{2}{*}{ Site } & \multirow{2}{*}{ Date } & \multicolumn{4}{|c|}{ Total reads (millions) } & \multicolumn{3}{|c|}{ Number of contigs } & \multicolumn{2}{|c|}{ Virus } \\
\hline & & Raw & Filtered & Deduplicated & Mapped & Assembled & $>300$ bp & BLAST & $\begin{array}{l}\text { Reads } \\
\text { (millions) }\end{array}$ & $\%^{*}$ \\
\hline Bol & May 18 & 48 & 44 & 8 & 3 & 7,890 & 6,917 & 4,340 & 0.9 & 12 \\
\hline Bol & August 18 & 60 & 56 & 9 & 6 & 10,200 & 8,576 & 4,148 & 4 & 45 \\
\hline Bol & September18 & 155 & 131 & 21 & 14 & 16,145 & 14,635 & 5,416 & 7 & 33 \\
\hline $\mathrm{Bol}$ & December 18 & 88 & 80 & 15 & 11 & 20,339 & 15,185 & 6,608 & 8 & 52 \\
\hline Bol & January 19 & 111 & 109 & 20 & 16 & 10,044 & 8,840 & 3,087 & 15 & 77 \\
\hline Bol & March 19 & 71 & 66 & 12 & 10 & 9,280 & 8,233 & 3,173 & 6 & 54 \\
\hline Bol & April 19 & 69 & 65 & 12 & 9 & 18,067 & 13,937 & 6,332 & 4 & 30 \\
\hline Bol & June 19 & 86 & 64 & 11 & 9 & 10,266 & 7,548 & 2,968 & 2 & 19 \\
\hline Bol & October 19 & 47 & 36 & 7 & 5 & 12,836 & 10,215 & 4,330 & 2 & 27 \\
\hline Bol & November 19 & 44 & 32 & 6 & 4 & 12,554 & 10,751 & 4,368 & 2 & 26 \\
\hline Bol & December 19 & 42 & 27 & 5 & 4 & 9,287 & 8,173 & 3,540 & 0.8 & 18 \\
\hline Bol & January 20 & 129 & 102 & 18 & 15 & 18,929 & 14,789 & 6,062 & 8 & 42 \\
\hline Bol & February 20 & 177 & 95 & 14 & 12 & 11,410 & 8,487 & 3,822 & 2 & 12 \\
\hline Mou & May 18 & 63 & 55 & 9 & 6 & 8,919 & 8,068 & 3,982 & 3 & 28 \\
\hline Mou & July 18 & 131 & 119 & 19 & 14 & 14,143 & 12,954 & 4,972 & 9 & 50 \\
\hline Mou & April19 & 70 & 62 & 12 & 9 & 15,763 & 12,640 & 5,370 & 4 & 38 \\
\hline Mou & June 19 & 67 & 57 & 11 & 8 & 12,655 & 10,061 & 3,737 & 4 & 40 \\
\hline Mou & October 19 & 78 & 68 & 12 & 9 & 18,287 & 14,316 & 5,776 & 4 & 35 \\
\hline Mou & November 19 & 51 & 37 & 7 & 5 & 12,444 & 10,060 & 4,407 & 1 & 21 \\
\hline Mou & December 19 & 152 & 89 & 14 & 11 & 14,108 & 11,406 & 4,847 & 3 & 24 \\
\hline Mou & January 20 & 87 & 82 & 15 & 11 & 15,704 & 11,940 & 4,046 & 5 & 31 \\
\hline Mou & February 20 & 90 & 54 & 9 & 7 & 9,475 & 7,552 & 3,145 & 2 & 19 \\
\hline
\end{tabular}

"The percentage was calculated by dividing the viral reads by the deduplicated reads (and $x 100$ ). 


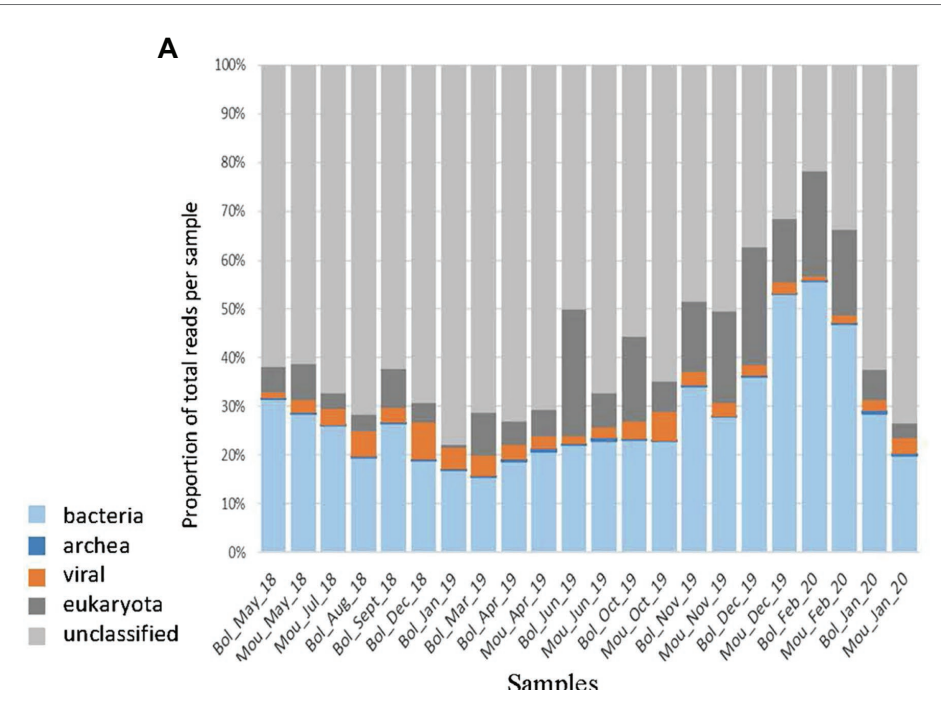

B

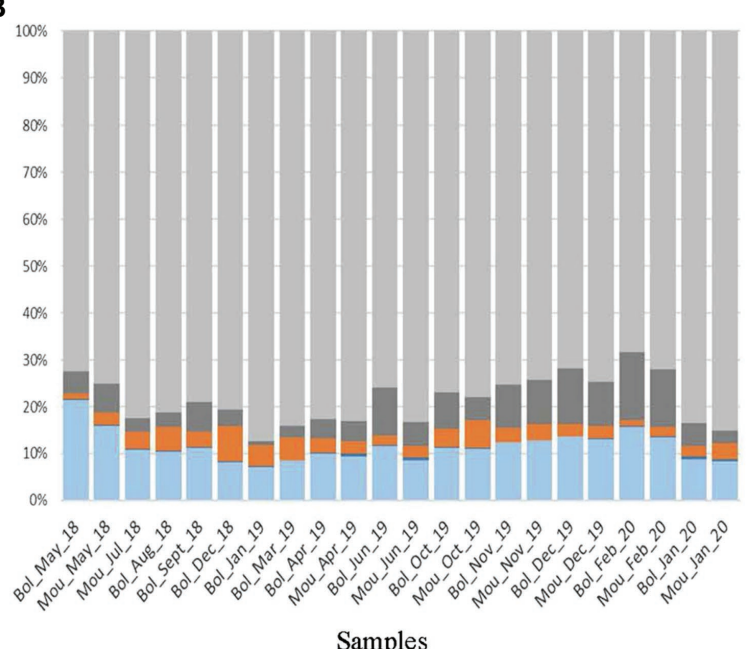

FIGURE 2 | Taxonomic assignation using Kaiju. The total reads obtained after filtering (A) or after deduplication (B) were reported for the different kingdoms such as bacteria (light blue), archea (dark blue), viral (orange), eukaryota (dark grey), and unclassified (light grey).

12 to $54 \%$, with only one sample reaching a higher percentage (77\%; Table 2). These viral reads were further identified and classified into different viral families based on BLASTn/ DIAMOND results (Figure 3). All samples displayed similar distribution of the reads regarding the different families selected here. As expected from our protocol for library preparation (DNAse treatment and enrichment in viruses from vertebrates), few reads mapped to phage families $(0.03 \%)$ or DNA viruses $(0.6 \%)$, except for Circoviridae family that was frequently identified. These reads were not further analyzed as a bias may exist, and the purpose of this study was to identify RNA viruses. The most abundant contigs belonged to unclassified riboviruses ( $96.6 \%$ of viral reads). Viral families infecting vertebrates or invertebrates hosts (Nodaviridae) or plants (Luteoviridae - now Solemoviridae, Tombusviridae) were also frequently detected (Figure 3). Contigs related to a rhabdovirus previously detected in a sample from an amphibian were present in samples collected at the end study but (December 2019 to February 2020). Reads mapping to mammalian RNA viruses were less frequent, the Astroviridae family being the most frequently recognized. No clear difference was observed between the two sampling sites or over the studied period.

\section{Identification Vertebrate RNA Virus Sequences That May Infect Humans}

Viral contigs longer than 300 bases, with at least five reads mapping on the same area and an E-value threshold of $10^{-15}$ for the BLAST, were selected to identify viral families that may be of interest for human disease. This strategy was used to identify sequences belonging to Astroviridae, Caliciviridae, Hepeviridae, Picobirnaviridae, and Picornaviridae families. Using these criteria, selected contigs were identified using either BLASTn or DIAMOND, and the host corresponding to the best BLAST match was considered (Figure 4). Sequences identified as belonging to the Picobirnaviridae family showed the greatest host diversity with about the same proportion of sequences related to strains infecting bats or other mammal, and few sequences related to strains infecting humans or unspecified hosts. Sequences identified as belonging to Caliciviridae or Picornaviridae families were mainly related to strains detected in bat samples, while many sequences identified as Astroviridae were not clearly related to any host. Sequences identified as Hepeviridae were almost all related to strains detected in sponge samples (data not shown).

\section{Astroviridae}

Contigs identified as belonging to the Astroviridae family were the most abundant with 841 sequences obtained representing 996,887 reads. A large number of these contigs (620) were similar to sequences previously identified as bastrovirus detected in sewage samples in Brazil (dos Anjos et al., 2017), including four sequences longer than 4,000 nucleotides (Table 3). One hundred seventeen contigs from 20 samples were found close to a bastro-like virus detected in stools of Vietnamese bats. Three sequences (2,311-5,030 bases) distantly related to non-structural polyprotein of a porcine bastrovirus were detected in three samples from both sites (Table 3). Regarding astrovirus, 85 sequences, of which 65 sequences ranging from 1,061 to 7,084 bases, were related to a sequence detected in a soil sample in the United States. Of note, the longest contig obtained (7,084 nucleotides) mapped only partially (1/3; Table 3$)$. Two sequences were found very similar to an astrovirus infecting a bird (European roller).

Phylogenetic trees based on the RdRp (Figure 5A) and capsid proteins (Figure 5B) confirmed that these bastrovirus sequences clustered together in one clade with sequences identified in Brazil or Vietnam. 


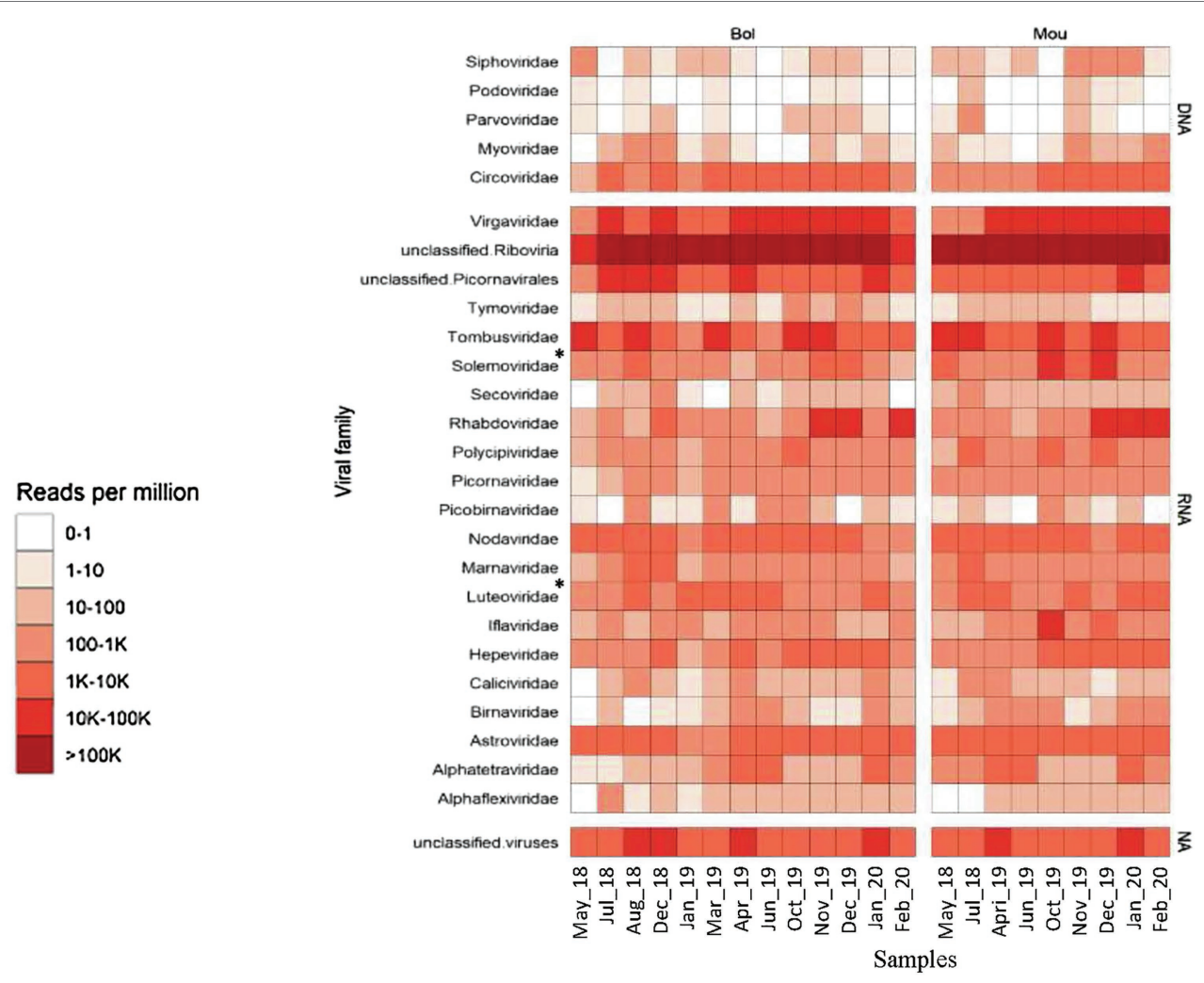

FIGURE 3 | Heatmap of the viral diversity. The read abundance after quality control and deduplication is presented for the two sites (Bol and Mou). Color gradients represent normalized relative abundance of reads expressed in reads per million (RPM) for each sample belonging to taxonomic groups indicated, with the top five rows corresponding to DNA viruses, the last row to unclassified viruses and middle rows to RNA viruses. *Since 2021, Luteoviridae was assigned to Solemoviridae.

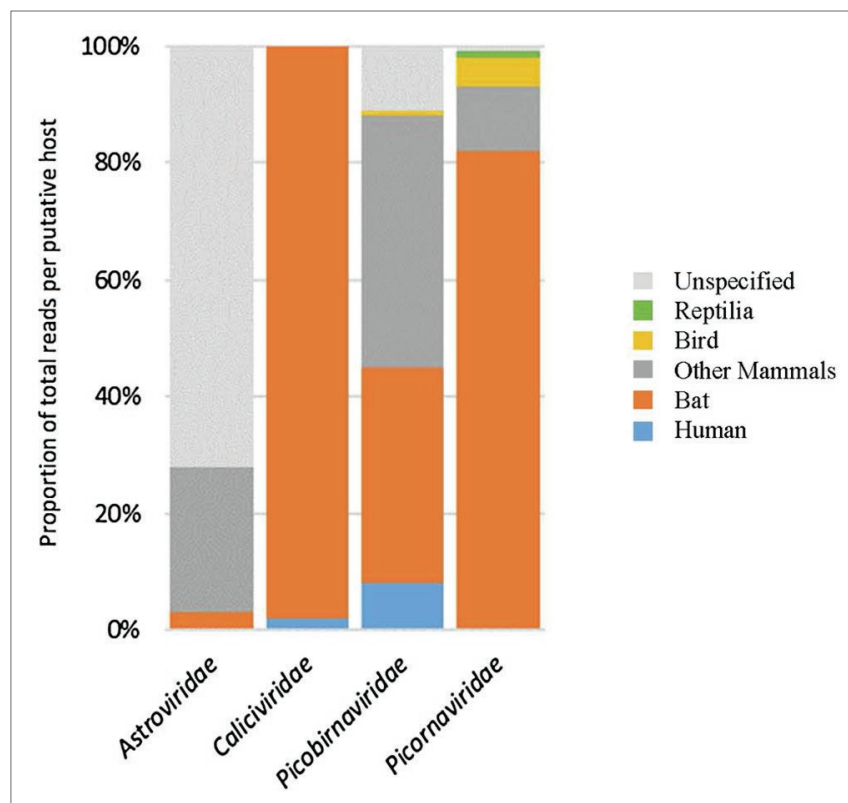

FIGURE 4 | Overview of the host diversity and reads identified as belonging to four viral families. For each family the number of reads was expressed in \% referring to the host potentially infected. Only the most abundant assigned reads are presented here, regarding their capacity to infect humans (blue), bats (orange), birds (yellow), other mammals (dark grey), and unspecified hosts (light grey).

\section{Caliciviridae}

Initially, 21 of the 22 selected samples were positive for human noroviruses as detected by RT-PCR. Only 64 contigs (352-1,833 bases) were assigned as Caliciviridae. Importantly, three of them were very likely of human origin: two contigs shared around 96\% nucleotide identity with a human norovirus GII.6 strain detected in a stool from China, and a third contig displayed $97 \%$ nucleotide identity with a human norovirus detected in Japan (Table 4). Several Caliciviridae contigs showed similarities with bat caliciviruses, five were related to the bat calicivirus A10 (Table 4). The longest Caliciviridae contigs $(1,026-1,833$ bases) were close to a sequence identified in a tricolored bat (Perimyotis subflavus) in the United States.

\section{Picobirnaviridae}

A large number of reads $(21,958)$ were identified as belonging to the Picobirnaviridae family. These reads were realigned on 45 contigs of $354-2,492$ base pairs identified as close to algae, human, bat, and monkey picobirnavirus strains. Eight contigs ranging from 391 to 615 base pairs were identified as closely related to human picobirnavirus sequences. Among these, five contigs, detected in Bol site, exhibited high nucleotide homology with a virus detected in human stool in India (Table 5). Other contigs were also related to human strains, one being previously detected in Cameroonian stool. Phylogenetic analysis of two 
TABLE 3 | Astroviridae contigs identified in Sanaga clams.

\begin{tabular}{|c|c|c|c|c|c|c|c|c|c|}
\hline Site & Date & $\begin{array}{l}\text { Accession } \\
\text { no." }\end{array}$ & $\begin{array}{l}\text { Length } \\
\text { (nt) }\end{array}$ & $\begin{array}{l}\text { Mapped } \\
\text { reads }\end{array}$ & $\begin{array}{l}\text { Nucleotide } \\
\text { match }\end{array}$ & $\begin{array}{l}\text { Identity } \\
(\%)\end{array}$ & E-value & Name & $\begin{array}{c}\text { Ref. } \\
\text { sequence }\end{array}$ \\
\hline Bol & September 18 & MW924352 & 5,823 & 27,103 & 4,221 & 87.6 & 0 & Bastrovirus & ASM79505.1 \\
\hline Bol & September 18 & MW924354 & 5,728 & 17,434 & 4,221 & 95.4 & 0 & & \\
\hline Mou & July 18 & MW924355 & 4,312 & 6,571 & 2,901 & 94.5 & 0 & & \\
\hline Mou & July 18 & MW924356 & 2,390 & 8,659 & 972 & 92.9 & $9.6 \times 10^{-164}$ & & ASM79506.1 \\
\hline Mou & July 18 & MW924359 & 1,097 & 1,161 & $1,097^{\#}$ & 79.8 & 0 & & \\
\hline Bol & December 18 & MZ182250 & 389 & 99 & $376^{\#}$ & 84.0 & $1.7 \times 10^{-95}$ & $\begin{array}{l}\text { Bastrovirus-like virus, } \\
\text { US-Culex }\end{array}$ & NC_040647.1 \\
\hline Bol & August 18 & MZ182271 & 5,030 & 1,874 & 1,044 & 29.0 & $1.2 \times 10^{-27}$ & Bastrovirus & QDA77022.1 \\
\hline Bol & February 20 & MZ182272 & 3,325 & 70,746 & 1,044 & 29.0 & $8.1 \times 10^{-28}$ & US-Porc & \\
\hline Mou & May 18 & MZ291967 & 2,311 & 7,679 & 1,062 & 27.7 & $7.4 \times 10^{-28}$ & & \\
\hline Bol & October 19 & MZ291968 & 404 & 36 & 390 & 56.9 & $3.4 \times 10^{-37}$ & Astrovirus & QBZ38214.1 \\
\hline
\end{tabular}

"This accession number corresponds to the sequence identified in this study and deposited in Genbank. Contig identification was done using DIAMOND except for some sequences noted with" for which BLASTn was used. Sequence identity is expressed as a percentage. E-value is from the BLAST tool.

of them based on RdRp sequences revealed clustering of both in genogroup I (Figure 6).

Beside human picobirnavirus, three contigs displayed similarities with a simian strain identified in gorilla stool in Democratic Republic of Congo and three contigs with strains detected in macaques (Duraisamy et al., 2018). Two contigs of 2,023-2,029 base pairs, detected in both sites in October 2019, were distantly related to a picobirna-like virus isolated from Cameroonian bats (Eidolon helvum; Yinda et al., 2018). Phylogenetic analysis of the longest one, based on RdRp sequences, showed clustering in a clade of uncharacterized picobirna-like viruses that use an alternative invertebrate mitochondrial genetic code (Figure 6).

\section{Picornaviridae}

Thirty-eight contigs gathering 69,517 reads, with sizes ranging from 353 to 4,631 bases, were identified as belonging to the Picornaviridae family. A short contig of 353 bases genetically close to the genogroup $\mathrm{V}$ of the simian hepatitis $\mathrm{A}$ virus was identified in a sample collected in Bol in October 2019 (Table 6). This sequence shared a high nucleotide identity (91.7\%) with the capsid gene of a simian strain detected in water samples in South Africa (Rachida et al., 2016). We previously sequenced a similar strain in a Sanaga clam sample collected in this area, albeit, with only $18 \%$ sequence overlap (Bonny et al., 2020).

With the exception of one contig displaying $76 \%$ aminoacid identity with its best DIAMOND match, the other contigs were more distantly related (26-38\% amino-acid identity) to Picornaviridae sequences from the database. However, e-values remained in the acceptable range. Contigs related to bat picornavirus were the most abundant (42\%) and were present in $68 \%$ of samples. With sizes ranging from 382 to 4,631 bases, most of them were closest at the protein level to the
Washington Bat picornavirus strain (Table 6). Other contigs were identified as being close to porcine picornavirus (453-1,220 bases) with similarities to sapelovirus A, sapelovirus 1 or kobuvirus, and few of them to chicken megrivirus.

\section{Hepeviridae}

A large number of contigs (188) of various lengths (300-8,223 nucleotides) representing 390,728 reads were identified as belonging to the Hepeviridae family. Most of them (92\%) were identified as close to sponge hepe-like virus. Only four contigs were related to mammalian viral sequences (Table 7): two were close to sequences detected in swine feces and the two others to a strain detected in mice.

\section{DISCUSSION}

The current COVID-19 pandemic highlights the importance of monitoring the possible transmission of viruses from wildlife to human. For this, the main challenge rests in detecting the viruses infecting diverse animal populations in their natural reservoirs and to predict their zoonotic potential (Osterhauss et al., 2020). Here, we propose to use clams as sentinels to detect potential environmental contamination with animal or human viruses, provided that identified sequences have been adequately related to their hosts. Clams, as other bivalve molluscan shellfish, filter large volumes of water. Furthermore, as benthic animals, they also are in close contact with sedimented particles such as the one originating from feces. In areas where collecting representative water samples is difficult, bivalve mollusks sampling may be a good alternative.

Preserving all viral genetic diversity presents in a sample is not possible yet since protocols used to date include steps 


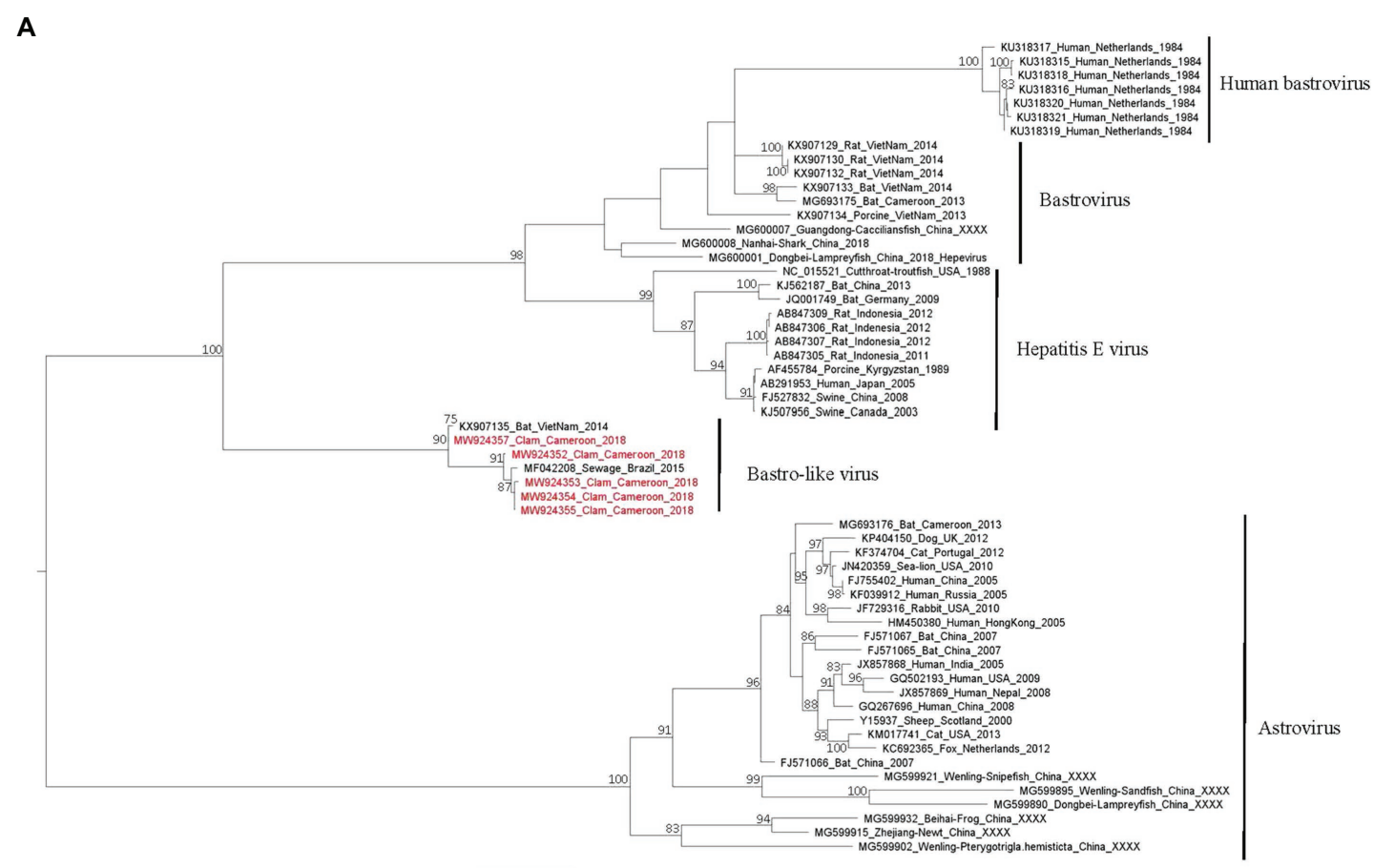

B

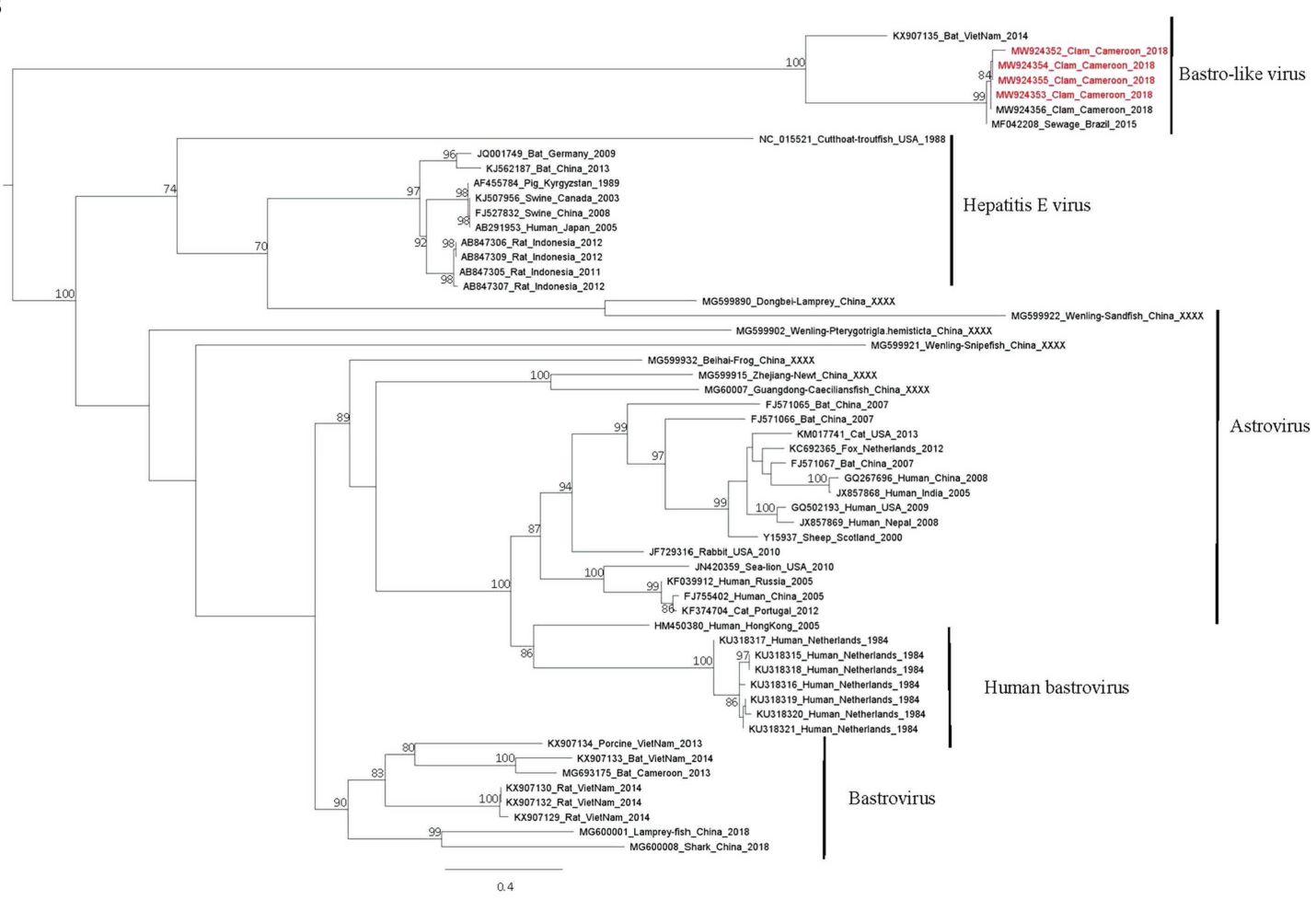

FIGURE 5 | Phylogenetic trees of the Astroviridae family based on Rdrp (A) and capsid (B) proteins. Reference sequences were selected for the main genus of the Astroviridae family, sequences identified in this study are written in red. The tree was constructed using IQtree with the $L G+I+G 4$ substitution model for the RdRp and the LG+G4 model for the capsid and ultrafast bootstrap analysis. Trees were mid-rooted using FigTree and boostrap values greater than $70 \%$ are shown.

that may favor the selection of certain types of viral particles. These can be, for example, (1) sampling bias, as collecting samples from the environment may be source of variations,
(2) concentration methods that may eliminate some viruses of interest or increase the presence of inhibitors that may impact the efficacy of enzymes used for the molecular detection, 
TABLE 4 | Caliciviridae sequences identified in Sanaga clams.

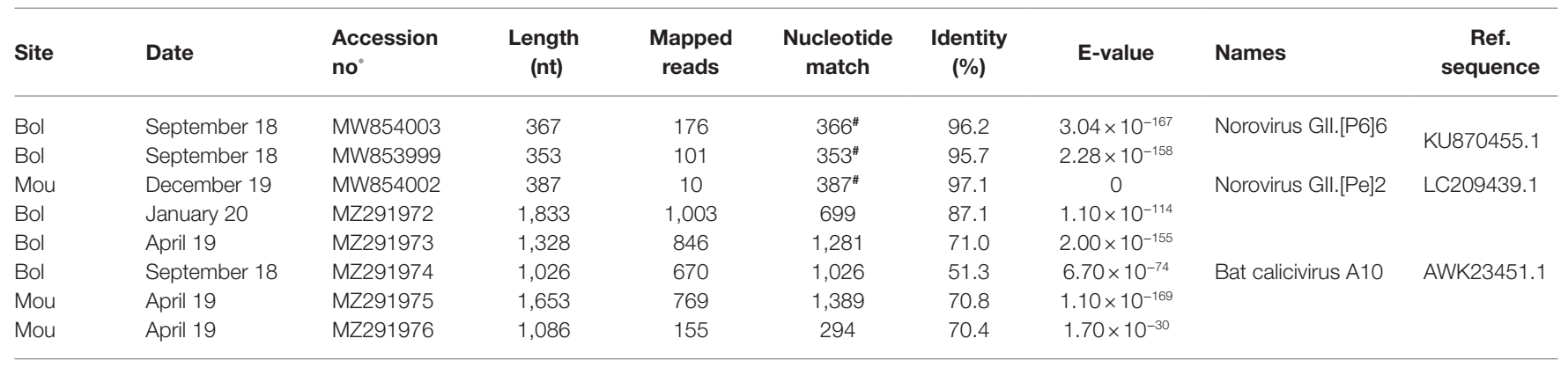

"The accession number corresponds to the sequence identified in this study and deposited in Genbank. Contig identification was done using Diamonds except for some sequences noted with " for which BLASTn was used. Sequence identity is expressed as a percentage. E-value is from the BLAST tool.

TABLE 5 | Picobirnaviridae sequences identified in Sanaga clams.

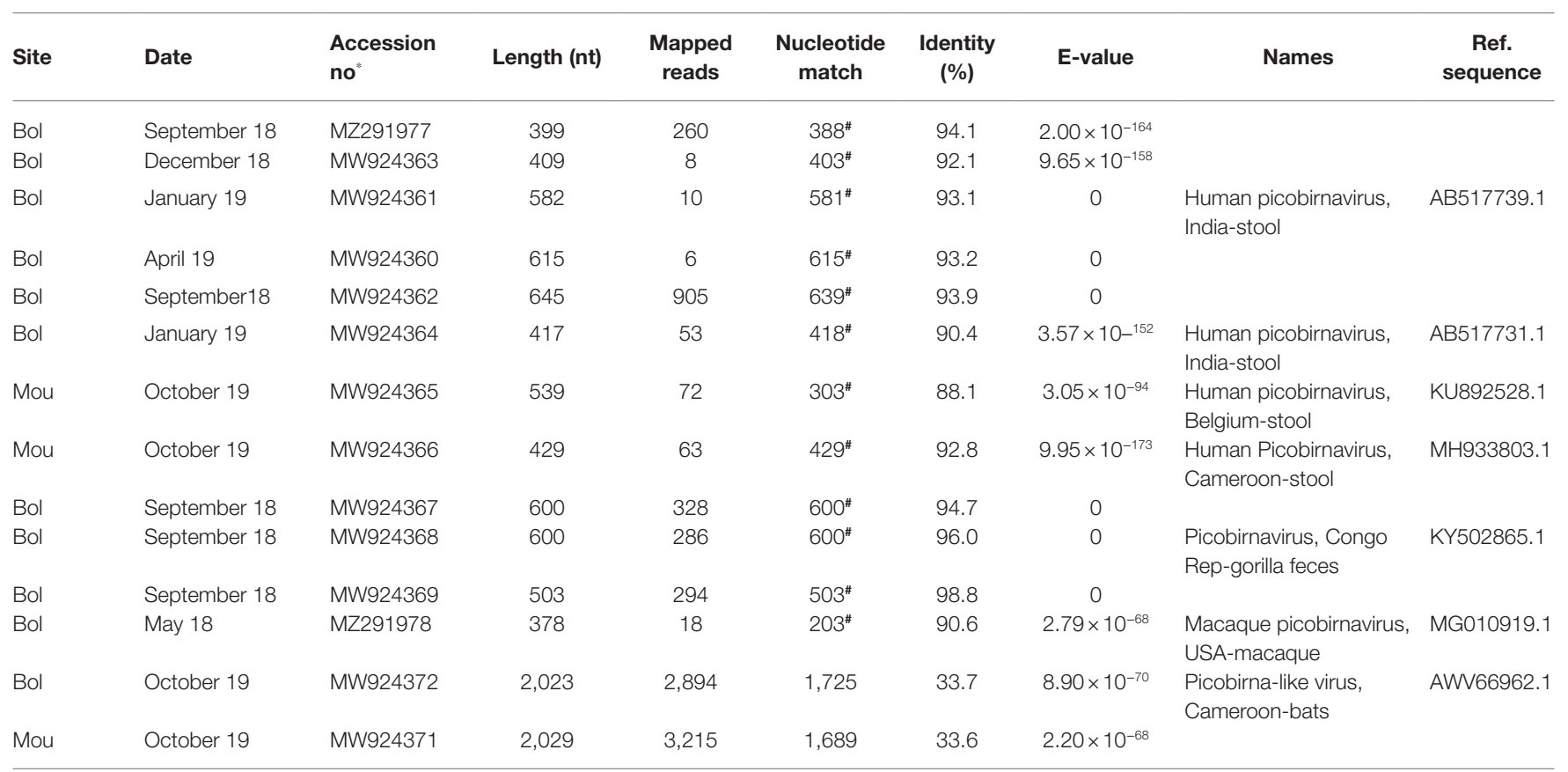

"This accession number corresponds to the sequence identified in this study and deposited in Genbank. Contig identification was done using Diamond except for some sequences noted with " for which BLASTn was used. Sequence identity is expressed as a percentage. E-value is from the BLAST tool.

(3) nucleic acid extractions and subsequent library preparation including DNAse or RNAse treatments, or (4) at the last step, the bioinformatic pipeline. Furthermore, searching for viruses with a short RNA genome present in low concentrations adds difficulties. A number of different approaches may help to solve some of these issues (Briese et al., 2015; Conceicao-Neto et al., 2015; Kim et al., 2017; Desdouits et al., 2020). In our previous work, we proposed methodological approaches that aimed to eliminate as much as possible bacteria, shellfish tissues, or free DNA or RNA (Strubbia et al., 2020), to focus on RNA viruses. This likely explains the low amount of DNA and phages viruses reads in our study unlike agnostic metagenomic studies where these viruses constituted the largest viral fraction (Bačnik et al., 2020; Nieuwenhuijse et al., 2020). We also performed technical triplicates of cDNA synthesis and libraries as we observed that it increases the odds to recover sequences from viruses present at low concentrations (Strubbia et al., 2020). Generating technical or biological replicates help to increase virus diversity (Kim et al., 2017). Furthermore, we used an enrichment step based on capture of cDNA from vertebrate viruses using the VirCapSeq-VERT assay during libraries preparation (Wylie et al., 2015; Strubbia et al., 2019). Finally, considering the low concentrations of norovirus and hepatitis A virus as measured by quantitative PCR, we selected a NovaSeq run to increase the depth per sample and thus increase the number of viral reads. As anticipated, we observed that viral reads remained a minority among the huge amount of reads that were obtained. These very large and diverse datasets resulted 


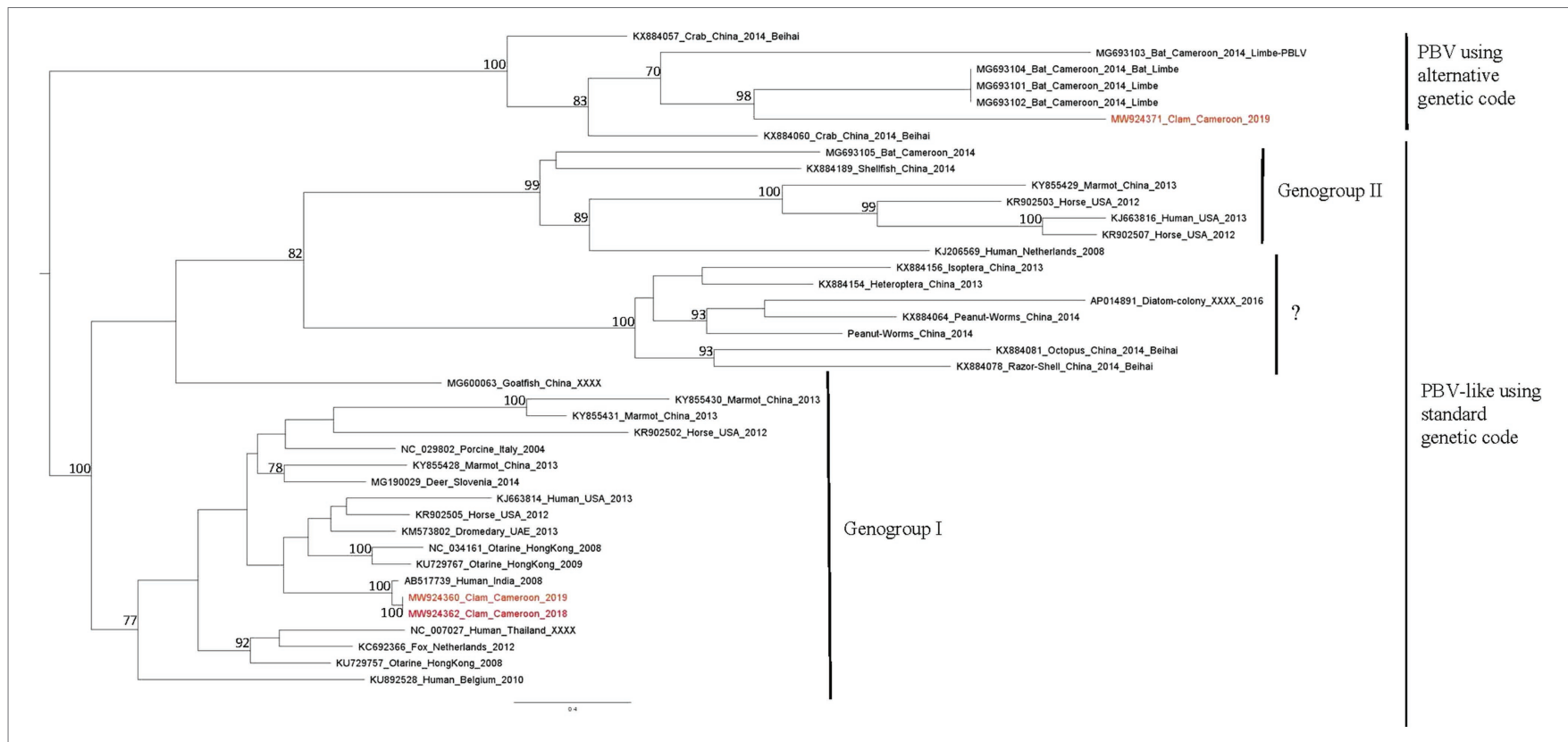

FIGURE 6 | Phylogenetic tree of RdRp sequences of picorbirnavirus. Reference sequences were selected to represent the three main clusters of picobirnavirus, sequences identified in this study are written in red. The tree was constructed using the Best-fit rtREV+F $+\mathrm{G} 4$ chosen according to BIC, boostrap values greater than $70 \%$ are shown.

in long, difficult, and sometimes impossible de novo assembly. Read deduplication has been shown to allow a faster and easier assembly by removing repeated reads, mostly generated by the amplification required to reach minimal DNA amounts for sequencing (Nieuwenhuijse et al., 2020). Here, this bioinformatic treatment considerably reduced fractions of bacteria and eukaryote reads, increased the number of unclassified reads, while fractions of viral reads remained stable in all samples. After deduplication, de novo assembly yielded assembled contigs for all samples, with a reduced computing time.

Another major issue in the metagenomic analysis of environmental samples is the presence of a large amount of unclassified sequences referred to as dark matter, formally defined as any nucleotide sequence that cannot be taxonomically assigned by alignment to any reference nucleotide or amino acid sequence (Krishnamurthy and Wang, 2017; Sunagawa et al., 2020). Samples derived from complex mixtures like those from mammalian guts or environmental samples are known to include novel organisms not described in reference sets or otherwise undocumented (Connor et al., 2019). Characterization of the viral fraction is especially difficult as only a small portion of Earth's viral diversity has been identified and made available in reference sets (Connor et al., 2019; Plyusnin et al., 2020; Sunagawa et al., 2020). In our study, up to $87 \%$ of reads could not be identified, and $96 \%$ of RNA viral reads could not be assigned to any viral family. Such observations previously reported in other studies, confirmed that although advances in high-throughput sequencing expand the boundaries of viral sequence space, some developments are needed to increase viral annotations (Bačnik et al., 2020; Nieuwenhuijse et al., 2020; Dance, 2021).
In any case, it is important to continue improving bioinformatic pipelines and databases to be able to screen rapidly metagenomic data and fully identify the organisms in a sample (Nieuwenhuijse et al., 2020; Plyusnin et al., 2020; Dance, 2021).

To reliably identify the very diverse sequences obtained through de novo assembly, each contig was identified by BLAST considering the length of overlap with the database sequence match, the numbers of reads and the BLAST E-value. The viral diversity in our study is described for all samples together rather than comparing the two sites or the season as the same sequences were detected in both sites and considering that the number of samples or the duration of the study may not be long enough to be able to detect some differences.

Following a one-health approach, we focused on the identification of RNA mammalian viral sequences, and especially on the main viral families potentially related to human health and known to be present in human or animal excreta such as Astroviridae, Caliciviridae, Hepeviridae, Picobirnaviridae, and Picornaviridae. Of note, since we rely on the sequence only, host assignation in our study is based on the host identified for the best BLAST match, which can be quite distantly related to our sequences. We grouped putative hosts into large families like humans, swine, or bats (Figure 3), but this information should be considered as indicative, and not definitive, as some sequences may have been detected in samples that are not their replicating host, and hosts may be misidentified in databases (Cobbin et al., 2021).

Few sequences from human viruses were identified compared to the diversity of other sequences detected. This may be due to the low human population density and other environmental parameters such as currents or sewage disposal that were 


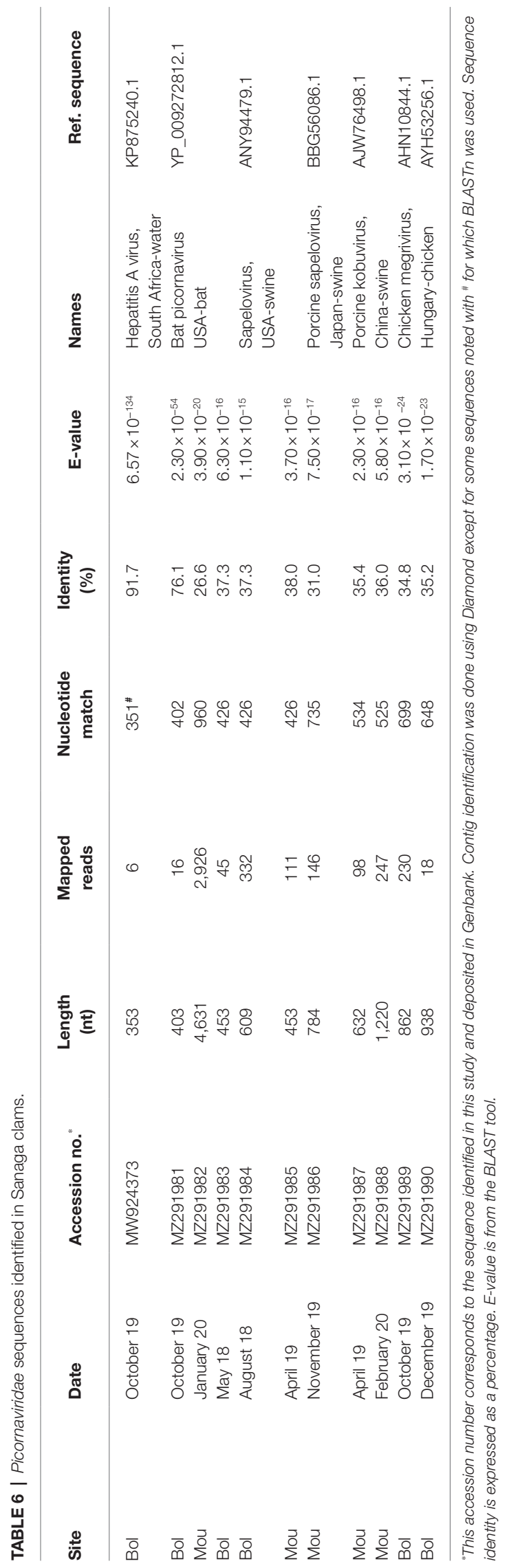

not investigated here. As mentioned above, all but one of the samples selected for the metagenomic analysis were detected positive for norovirus by real-time RT-PCR. Despite this first screening results, very few related sequences were obtained, meaning that the sensitivity of the metagenomics technique needs to be further improved. In a previous study, we were able to identify a large diversity of norovirus in oysters displaying similar concentrations as these clam samples (Strubbia et al., 2019). In the current study, the enrichment step approach may have failed to catch all strains present in the nucleic acid extracts, or the large diversity of sequences obtained could have prevented contig assembly. Among the $40 \%$ of reads identified as norovirus and not assembled into contigs, most of them matched to the A-poly tail, suggesting that contig assembly was performant on this virus. Data on norovirus prevalence in this area are still scarce and to our knowledge only one study was published. Based on the analysis of a large panel of stool from all age groups, few sequences of norovirus were identified compared to a much higher diversity of sapovirus sequences that were not detected in our study (Yinda et al., 2019). This observation highlights the need to get more data on molecular epidemiology of viral pathogens in the local population. At variance to norovirus, many picobirnavirus sequences were identified but, despite several attempts, only short contigs could be assembled. Described as opportunistic viruses, their role in the occurrence of gastroenteritis in humans is still poorly studied and the true host remains enigmatic (Ghosh and Malik, 2021). However, a large diversity of picobirnavirus sequences was reported in the gut virome analysis of Cameroonians, most of them being detected in age groups above 20 (Yinda et al., 2019). The authors also mentioned the characterization of picobirna-like virus $99 \%$ identical to Cameroonian bat strain picorbirnalike viruses.

Most of the contigs identified in this study as mammalian viruses were most closely related to viruses identified in bats. We characterized two sequences longer than 2,000 nucleotides showing similarities with a picobirnavirus strain previously described in Cameroonian fruit bats (Yinda et al., 2018). Moreover a bastrolike-virus sequence was closely related to a strain previously detected in bat samples in VietNam but also in Cameroon; however, with no insurance that the bats were infected (Yinda et al., 2018). Several sequences related to bat calicivirus were identified in up to five samples. All these contigs showed the best homologies with the same strain identified in a bat stool collected the United States (Kocher et al., 2018). The capsid gene of this bat calicivirus A10, was closely related to norovirus genogroup $\mathrm{V}(\mathrm{GV})$ and phylogenetically related to recoviruses. This strain displayed similarities with human noroviruses in terms of binding pattern to human histo-blood group antigens, suggesting the potential of this virus to overcome a significant barrier to cross-species transmission (Kocher et al., 2018). The potential zoonotic transmission is a long-standing question regarding norovirus, with scarce data reported so far, highlighting the need to identify the potential reservoir (Villabruna et al., 2019). A bat picornavirus sequence was 
TABLE 7 | Hepeviridae sequences identified in Sanaga clams.

\begin{tabular}{|c|c|c|c|c|c|c|c|c|c|}
\hline Site & Date & $\begin{array}{c}\text { Accession } \\
\text { no }^{*}\end{array}$ & $\begin{array}{l}\text { Length } \\
\text { (nt) }\end{array}$ & $\begin{array}{l}\text { Mapped } \\
\text { reads }\end{array}$ & $\begin{array}{c}\text { Nucleotide } \\
\text { match }\end{array}$ & $\begin{array}{l}\text { Identity } \\
\text { (\%) }\end{array}$ & E-value & Names & $\begin{array}{c}\text { Ref. } \\
\text { sequence }\end{array}$ \\
\hline Bol & February 20 & MZ476780 & 586 & 49 & 498 & 85 & $7.40 \times 10^{-17}$ & Hepatitis E virus, China-swine & ACD44941.2 \\
\hline Bol & December 18 & MZ476781 & 572 & 46 & 570 & 100 & $1.30 \times 10^{-18}$ & Hepatitis E virus, China-rabbit & BAM68889.1 \\
\hline Bol & April 19 & MZ476782 & 558 & 34 & 498 & 89 & $1.10 \times 10^{-22}$ & & \\
\hline Mou & June 19 & MZ476783 & 611 & 96 & 537 & 88 & $4.50 \times 10^{-17}$ & Hepe-lIke virus, USA-mıce & AVVB14594.1 \\
\hline
\end{tabular}

"This accession number corresponds to the sequence identified in this study and deposited in Genbank. Contig identification was done using Diamond except for some sequences noted with" for which BLASTn was used. Sequence identity is expressed as a percentage. E-value is from the BLAST tool.

also identified in two samples. Taken together these observations suggest a repeated contamination of the studied area by bat feces. Bats are considered as a major reservoir of viruses, and several studies reported the characterization of viruses closely related to human viruses confirming their potential role for zoonotic events (Hardmeier et al., 2018; Yinda et al., 2018). Based on our study, we suggest to consider the environmental contamination by these animals as it may constitute a driver for human contamination (Gortazar et al., 2014; Kohl et al., 2021).

Among the animal virus sequences detected in this work, those infecting pigs were second in terms of diversity. We detected not only some sapelovirus and kobuvirus from the Picornaviridae family but also some bastrovirus, bastro-like virus, and some Hepatitis E virus. Considering the capacity of this virus to infect across species barriers, monitoring prevalence of this virus in pigs in the region may be of interest (Sooryanarain and Meng, 2019).

In a previous study conducted in this area, we sequenced a portion of the genome of a hepatitis A virus, related to genogroup $\mathrm{V}$ which include simian viruses, and most closely related to a potentially simian virus identified in surface waters (Bonny et al., 2020). We detected again this sequence and also some simian picobirnavirus sequences confirming the contamination of the area with macaque feces. The Sanaga river, a rural area, harbors a wildlife reserve (Figure 1) characterized by abundant and diverse fauna, including an important simian population. Zoonotic transmissions have been proven from non-human primates to humans for different viral families (Devaux et al., 2019; Di Cola et al., 2021). However, none of the human viral strains from Cameroon were related to apes in the study conducted in 2014 (Yinda et al., 2019).

Our approach considering the diversity of RNA virus sequences detected in this area may contribute to the surveillance of zoonotic events (Osterhauss et al., 2020; Williams et al., 2021). Indeed, despite a need for better sensitivity, we report here a method that succeeded to describe a large diversity of RNA viruses from clam samples. In addition to their role as a sentinel of the microbial environmental quality, shellfish constitute an easy source of proteins for local populations with low economic resources (Ajonina et al., 2005; Venugopal and Gopakumar, 2018). While the way of consumption (boiled and smoked) likely prevents foodborne transmission of these viruses, the first steps of opening the shell and collecting the raw flesh may be at risk for the workers. Thus, it is important to share information and provide some recommendation to local populations.

Metagenomic approaches are very promising tools to describe viral diversity and will be of particular interest for food and environmental samples analysis (Desdouits et al., 2020). It is especially relevant for shellfish analysis. Indeed, bivalve molluscan shellfish, a nutrient source for many populations, may also reflect microbial contamination shed by surrounding animal or humans. Being able to describe all viral contaminants in molluscan bivalve shellfish, whether considered as a food or environmental sentinel may contribute to the better understanding of some zoonotic transmission events and alert health authorities of possible emergence.

\section{DATA AVAILABILITY STATEMENT}

The datasets presented in this study can be found in online repositories. The names of the repository/repositories and accession number(s) can be found at: https://doi.org/10.12770/5abfae46-aca449ad-b1bc-4fe5a1ba4454.

\section{AUTHOR CONTRIBUTIONS}

$\mathrm{PB}$, JS, MD, and FG contributed to the conception and design of the study. $\mathrm{PB}$, JS, and $\mathrm{AB}$ performed the bio-info analysis. $\mathrm{PB}, \mathrm{JS}, \mathrm{AB}, \mathrm{MD}$, JE, and $\mathrm{FG}$ contributed to data interpretation and manuscript preparation. All authors contributed to the article and approved the submitted version.

\section{FUNDING}

This study was supported by the VEO European project (H2020, SC1-2019-874735). PB was supported by a founding from Ministry of Scientific Research and Innovation of Cameroon.

\section{ACKNOWLEDGMENTS}

This work benefited from equipment and services from iGenSeq core facility, at ICM (Institut Cerveau Moelle, Paris, France). We thank B. Oude Munnink and D. Nieuwenhuijse for helpful scientific discussion. 


\section{REFERENCES}

Ajonina, P. U., Ajonima, G., Jin, E., Mekongo, F., Ayisse, I., and Usongo, L. (2005). Gender roles and economics of exploitation, processing and marketing of bivalves and impacts on forest resources in the Sanaga Delta region of Douala-Edea wildlife reserve, Cameroon. Int. J. Sustain. Dev. World Ecol. 12, 161-172. doi: 10.1080/13504500509469627

Bačnik, K., Kutnjak, D., Pecman, A., Mehle, N., Žnidarič, M. T., Aguirre, I. A., et al. (2020). Viromics and infectivity analysis reveal the release of infective plant viruses from wastewater into the environment. Wat. Res. 177:115628. doi: $10.1016 /$ j.watres.2020.115628

Bonny, P., Desdouits, M., Schaeffer, J., Garry, P., Essia Nganag, J. J., and Le Guyader, F. S. (2020). Contamination of clams with human norovirus and a novel hepatitis A virus in Cameroon. Food Environ. Virol. 12, 274-277. doi: 10.1007/s12560-020-09432-2

Briese, T., Kapoor, A., Mishra, N., Jain, K., Kumar, A., Jabado, O. J., et al. (2015). Virome capture sequencing enables sensitive viral diagnosis and comprehensive virome analysis. MBio 6, e01491-e01415. doi: 10.1128/ mBio.01491-15

Buchfink, B., Xie, C., and Huson, D. H. (2015). Fast and sensitive protein alignment using diamond. Nat. Methods 12, 59-60. doi: 10.1038/nmeth. 3176

Camacho, C., Coulouris, G., Avagyan, V., Ma, N., Papadopoulos, J., Bealer, K., et al. (2009). BLAST+: architecture and applications. BMC Bioinformatics 10:421. doi: 10.1186/1471-2105-10-421

Cobbin, J., Charon, J., Harvey, E., Holmes, E. C., and Mahar, J. (2021). Current challenges to virus discovery by meta-transcriptomics. Curr. Opin. Virol. 51, 48-55. doi: 10.1016/j.coviro.2021.09.007

Conceicao-Neto, N., Zeller, M., Lefrere, H., de Bruyn, P., Beller, L., Deboutte, W., et al. (2015). Molecular approach to customise sample preparation procedures for viral metagenomics: a reproducible protocol for virome analysis. Sci. Rep. 5:16532. doi: 10.1038/srep16532

Connor, R., Brister, R., Buchmann, J. P., Deboutte, W., Edwards, R., Martí-Carreras, J., et al. (2019). NCBI's virus discovery Hackathon: engaging research communities to identify cloud infrastructure requirements. Genes 10:714. doi: 10.3390/genes10090714

Dance, A. (2021). The incredible diversity of viruses. Nature Rev. 595, 22-25.

Desdouits, M., de Graaf, M., Strubbia, S., Oude Munnink, B. B., Kroneman, A., Le Guyader, F. S., et al. (2020). Novel opportunities for NGS-based one health surveillance of foodborne viruses. One Health Outlook 2:14. doi: 10.1186/s42522-020-00015-6

Devaux, C. A., Mediannikov, O., Medkour, H., and Raoult, D. (2019). Infectious disease risk across the growing human-non human primate interface: a review of the evidence. Front. Public Health 7:305. doi: 10.3389/fpubh.2019.00305

Di Cola, G., Fantalli, A. X., Pisano, M. B., and Ré, V. E. (2021). Foodborne transmission of hepatitis A an hepatitis E viruses: a litterature review. Int. J. Food Microbiol. 338:10898986. doi: 10.1016/j.ijfoodmicro.2020.108986

dos Anjos, K., Nagata, T., and Melo, F. L. D. (2017). Complete genome sequence of a novel bastrovirus isolated from raw sewage. Genome Announc. 5, e01010-e01017. doi: 10.1128/genomeA.01010-17

Duraisamy, R., Akiana, J., and Mediannikov, Davoust B., O., Michelle, C., Robert, C. et al. (2018). Detection of novel RNA viruses from free-living gorillas, Republic of the Congo: genetic diversity of picobirnaviruses. Virus Genes 54: 256-271. doi: 10.1007/s11262-018-1543-6

Fu, L., Niu, B., Zhu, Z., Wu, S., and Li, W. (2012). CD-HIT: accelerated for clustering the next-generation sequencing data. Bioinformatics 28, 3150-3152. doi: 10.1093/bioinformatics/bts565

Ghosh, S., and Malik, Y. S. (2021). The true host/s of picobirnaviruses. Front. Vet. Sci. 7:615293. doi: 10.3389/fvets.2020.615293

Gortazar, C., Reperant, L. A., Kuiken, T., de la Fuente, J., Boadella, M., Martinez-Lopez, B., et al. (2014). Crossing the interspecies barriers: opening the door to zoonotic pathogens. Plos Pathog. 10:e1004129. doi: 10.1371/ journal.ppat.1004129

Hardmeier, I., Aeberhard, N., Qi, W., Schoenbaechler, K., Krattli, H., Hatt, J.M., et al. (2018). Metagenomic analysis of fecal and tissue samples from 18 endemic bat species in Switzerland revealed a diverse virus composition including potentially zoonotic viruses. PLoS One 16:e25534. doi: 10.1371/ journal.pone.0252534

ISO 15216-1. (2017). Microbiology of food and animal feed -horizontal method for determinaion of hepatitis A virus and norovirus in food using real-time RT-PCR, Part 1: method for quantification.

Kim, Y., van Bonn, W., Aw, T. G., and Rose, J. B. (2017). Aquarium viromes: viromes of human-managed aquatic systems. Front. Microbiol. 8:1231. doi: 10.3389/fmicb.2017.01231

Kocher, J. F., Lindesmith, L. C., Debbink, K., Beall, A., Mallory, M. L., Yount, B. L., et al. (2018). Bat caliciviruses and human noroviruses are antigenically similar and have overlapping histo-blood group antigen binding profiles. MBio 9, e00869-e00818. doi: 10.1128/mBio.00869-18

Kohl, C., Nitsche, A., and Kurth, A. (2021). Update on potentially zoonotic viruses of European bats. Vaccine 9, 690. doi: 10.3390/vaccines 9070690

Krishnamurthy, S. R., and Wang, D. (2017). Origins and challenges of viral dark matter. Virus Res. 239, 136-142. doi: 10.1016/j.virusres.2017. 02.002

La Rosa, G., Sanseverino, I., Della Libera, S., Laconelli, M., Ferrero, V. E. V., Barra Caracciolo, A., et al. (2017). The impact of anthropogenic pressure on the virological quality of water from the Tiber river, Italy. Lett. Appl. Microbiol. 65, 298-305. doi: 10.1111/lam.12774

Langmead, B., and Salzberg, S. (2012). Fast gapped-read aligment with bowtie 2. Nat. Methods 9, 357-359. doi: 10.1038/nmeth.1923

Metcalf, T., Melnick, J. L., and Estes, M. K. (1995). Environmental microbiology: from detection of virus in sewage and water by isolation to identification by molecular biology- a trip of over 50 years. Annu. Rev. Microbiol. 49, 461-487. doi: 10.1146/annurev.mi.49.100195.002333

Nieuwenhuijse, D., and Koopmans, M. P. G. (2017). Metagenomic sequencing for surveillance of food- and waterborne viral diseases. Front. Microbiol. 8:230. doi: $10.3389 /$ fmicb. 2017.00230

Nieuwenhuijse, D. F., Oude Munnink, B., Phan, M. V. T., the Global sewage Surveillance projet consortiumMunk, P., Venkatakrishnan, S., et al. (2020) Setting a baseline for global urban virome surveillance in sewage. Sci. Rep. 10:13748. doi: 10.1038/s41598-020-69869-0

Nooij, S., Schmitz, D., Vennema, H., Kroneman, A., and Koopmans, M. P. G. (2018). Overview of virus metagenomic classification methods and their biological applications. Front. Microbiol. 9:749. doi: 10.3389/fmicb.2018. 00749

Nurk, S., Meleshko, D., Korobeynikov, A., and Tevzner, P. A. (2017). metaSPAdes: a new versatile metagenomic assembler. Genome Res. 27, 824-834. doi: 10.1101/gr.213959.116

Osterhauss, A. D. M. E., Vanlangendonck, C., Barbeshi, M., Bruschle, M., Christensen, R., Daszak, P., et al. (2020). Make science evolve into a One Health approach to improve health and security: a white paper. One Health Outlook 2:6. doi: 10.1186/s42522-019-0009-7

Plyusnin, I., Kant, R., Jaaskelainen, A. J., Sironen, T., Holm, L., Vapalahti, O., et al. (2020). Novel NGS pipeline for virus discovery form a wide spectrum of hosts and sample types. Virus Evol. 6:veaa091. doi: 10.1093/ve/ veaa091

Polo, D., Lois, M., Fernandez-Nunez, M. T., and Romalde, J. L. (2021). Detection of SARS-CoV-2 RNA in bivalve mollusks and marine sediments. Sci. Total Environ. 786:147534. doi: 10.1016/j.scitotenv.2021.147534

Rachida, S., Matsapola, P. N., Wolfaardt, M., and Taylor, M. B. (2016). Genetic characterization of a novel hepatitis A virus strain in irrigation water in South Africa. J. Med. Virol. 88, 734-737. doi: 10.1002/jmv.24370

Sooryanarain, H., and Meng, X.-J. (2019). Hepatitis E virus: reasons for emergence in humans. Curr. Opin. Virol. 34, 10-17. doi: 10.1016/j.coviro.2018.11.006

Strubbia, S., Schaeffer, J., Besnard, A., Wacrenier, C., Le Mennec, C., Garry, P., et al. (2020). Metagenomic to evaluate norovirus genomic diversity in oysters: impact on hexamer selection and targeted capture-based enrichment. Int. J. Food Microbiol. 323:108588. doi: 10.1016/j.ijfoodmicro.2020.108588

Strubbia, S., Schaeffer, J., Oude Munnink, B. B., Besnard, A., Phan, M. V. T., Nieuwenhuijse, D. F., et al. (2019). Metavirome sequencing to evaluate norovirus diversity in sewage and related bioaccumulated oysters. Front. Microbiol. 10:3389. doi: 10.3389/fmicb.2019.02394

Sunagawa, S., Acina, S. G., Bork, P., Bowler, C., Coordinators, T. O., Eveillard, D., et al. (2020). Tara oceans: towards global ocean ecosystems biology. Nature Rev. Microbiol. 18. doi: 10.1038/s41579-020-0364-5 
Venugopal, V., and Gopakumar, K. (2018). Shellfish: nutritive value, health benefits, and consumer safety. Compr. Rev. Food Sci. Food Saf. 16, 1219-1242. doi: $10.1111 / 1541-4337.12312$

Villabruna, N., Koopmans, M. P. G., and de Graaf, M. (2019). Animals as reservoir for human norovirus. Viruses 11:478. doi: 10.3390/v11 050478

Williams, E. P., Sprill-Harrel, B. M., Taylor, M. K., Lee, J., Nywening, A. V., Yang, Z., et al. (2021). Common themes in zoonotic spillover and disease emergences: lessons learned from bat and rodent-borne RNA viruses. Viruses 13:1509. doi: 10.3390/v13081509

Wylie, T. N., Wylie, K. M., Herter, B. N., and Storch, G. A. (2015). Enhanced virome sequencing using targeted sequence capture. Genome Res. 25, 1910-1920. doi: $10.1101 /$ gr.191049.115

Yinda, C. K., Ghogomu, S. M., Conceicao-Neto, N., Beller, L., Deboutte, W., Vanhulle, E., et al. (2018). Cameroonian fruit bats harbor divergent viruses, including rotavirus $\mathrm{H}$, bastroviruses, and picobirnaviruses using an alternative genetic code. Virus Evol. 3:vey008. doi: 10.1093/ve/ vey008

Yinda, C. K., Vanhulle, E., Conceicao-Neto, N., Beller, L., Deboutte, W., Shi, C., et al. (2019). Gut microbiome of Cameroonians reveals high diversity of enteric viruses, including potential interspecies transmitted viruses. $m S$ phere 4, e00585-e00518. doi: 10.1128/mSphere.00585-18

Conflict of Interest: The authors declare that the research was conducted in the absence of any commercial or financial relationships that could be construed as a potential conflict of interest.

Publisher's Note: All claims expressed in this article are solely those of the authors and do not necessarily represent those of their affiliated organizations, or those of the publisher, the editors and the reviewers. Any product that may be evaluated in this article, or claim that may be made by its manufacturer, is not guaranteed or endorsed by the publisher.

Copyright (c) 2021 Bonny, Schaeffer, Besnard, Desdouits, Essia Ngang and Le Guyader. This is an open-access article distributed under the terms of the Creative Commons Attribution License (CC BY). The use, distribution or reproduction in other forums is permitted, provided the original author(s) and the copyright owner(s) are credited and that the original publication in this journal is cited, in accordance with accepted academic practice. No use, distribution or reproduction is permitted which does not comply with these terms. 